\title{
Journal of Sports Injuries and Medicine
}

\section{All Inside Meniscal Repair: Three Years Follow up}

\author{
Ginesin E, Sadia S, Peskin B D.
}

Department of Orthopedics, Rambam Health Care Campus, Haifa, Israel

"Corresponding author: Eyal Ginesin, Orthopedics department, Rambam health care campus, Haifa, Israel. Tel: +97247771298; Email: E_ginesin@rambam.health.gov.il

Citation: Ginesin E, Dreyfuss D, Stahl I, Peskin B (2017) All Inside Meniscal Repair: Three Years Follow up. Sports Injr Med: JSIMD-118. DOI: 10.29011/JSIMD-118/100018

Received Date: 1 June, 2017; Accepted Date: 30 October, 2017; Published Date: 6 November, 2017

\section{Introduction}

Meniscal repair was first done by Annandale in 1883. The meniscus was generally thought to be unimportant for the function of the knee. Tears in the meniscus typically were treated by complete excision of the meniscus. since 1968, when Jackson wrote, "The exact function of that structure (meniscus) is still a matter of some conjecture.", several researches that checked the knee function after a long follow-up following menisectomy have shown it to be deleterious to the joint surface and degenerative changes were directly proportional to the amount of meniscus removed [1-2], since then; there has been an increasing awareness of the importance of preserving the meniscus for load transmission, stability, and knee function. Techniques of meniscal repair have evolved from open, to semi open, to arthroscopic repair, and devices to facilitate each have evolved as well. The first arthroscopic repair was performed by Hiroshi Ikeuchi in 1969, Ikeuchi's report led to significant interest in techniques to preserve the meniscus [2]. The gold standard for meniscus repair has been-for many years- the inside-out technique. This technique has produced healing rates of 60 to $80 \%$ with isolated repairs and roughly $90 \%$ with repairs in conjunction with Anterior Cruciate Ligament (ACL) reconstruction $[1,3,4]$. The second generation of techniques was introduced by Russel Warren in 1985.This outside-in technique was designed to decrease the risk of neurovascular injury, especially to the peroneal nerve during lateral repair and saphenous nerve in medial repairs [5]. The long operating time and the need of creating additional posterior medial and posterior lateral incisions, a third generation of all-inside meniscal repair devices has been created. The first generation of all-inside meniscal repair was first described by Morgan [6,7], Mulhollan [8], and Reigel et al. [9] to repair posterior horn tears, it involved curved suture-hooks that were passed in the back of the knee through additional cannulas [10]. The T -Fix (Smith \& Nephew, Andover, MA) was the first widely accepted, all-inside device that could be inserted via standard anterior arthroscopic portals. The T-Fix required placement of two separate sutures across a tear. However, the 2-0 no absorbable suture functioned to prevent tear displacement and resulted in moderate success. The spin-off of the T- fix success involved various absorbable devices, including arrows, darts, screws, fasteners, and other devices that anchored the torn meniscus to the periphery, but included some way to better compress the tear. The meniscal arrow (Bionx, Blue Bell, PA) was probably the most popular, as it was basically the first of these devices. The common concern with all of these devices was the risk of articular cartilage damage resulting from an abrasion by the rigid implant [10].

The newest fourth generation of all-inside meniscal repair devices includes two new implants that incorporate a combination of suture, slip knot and a peripheral anchor [11]. The FasT-Fix (Smith \& Nephew) involves two of the T-Fix anchors on a single inserter. The anchors are separately placed at the rim of the meniscus through the tear, and then a slip knot is tensioned across the tear.

One of the most used second generations was the Rapid-Loc meniscal repair device (Debuy-Mitek, Westwood, MA). It involves a single backstop that is deployed on the outer rim of the meniscus, it consists of 3 elements: a "Top Hat" and a bar, and a suture made of No.2 Ethibond. This device is inserted into the meniscus with the use of a gun that fires the bar through the inner portion of the meniscus tear, on through the peripheral meniscal rim, and into the joint capsule. The purpose of our study was to evaluate the results of repair using the Rapidloc device.

\section{Methods}

During the years 2007-2008, we performed meniscal repair in 22 patients. All of the procedures were done by one surgeon (B.P.) at Rambam health campus, and in all the cases we used the Rapidloc all-inside meniscal repair device. The patients were evaluated by the subjective and objective International Knee Documentation Committee (IKDC) and Lysholm functional knee score. The period for follow up was one year and 3 years, the patients were assessed during this period at three points of time, preoperatively, and then postoperatively at 3 months, one year and 3 years. The criteria for repair, and thus inclusion in the study, included patients with unstable peripheral tear of the meniscus in red-red or red-white zones (Copper zone 1-2), minimal length of $10 \mathrm{~mm}$, who 
underwent meniscal repair using the Rapidloc device only. Patients who had Anterior Cruciate Ligament (ACL) instability, stability were restored with a concurrent reconstruction of the ACL. We accepted $P$ values of less than 0.5 as statistically significant based on Paired and Mann-Whitney U tests.

\section{Results}

The study population included 22 , completed the one-year follow up, in which we performed 22 meniscal repairs, and 13 completed the 3 years follow up. The average age was 22 (between 16 and 31), and the follow up was for one year. There were 18 males $(82 \%)$ and 4 females. 20 tears were at the medial meniscus and 2 at the lateral, 18 of the medial meniscus tears were in the posterior horn and 2 in the meniscus body and another in the anterior horn. All patients had tears in Cooper zones 1 and 2. An average of 2.5 sutures was used to repair the meniscal tear (between 1 and 4). 9 (41\%) underwent concurrent ACL reconstruction, in 13 (59\%) the meniscal tear was an isolated injury. Tear length average was 2.5 $\mathrm{cm}$ (between 1 and $5 \mathrm{~cm}$ ).

The success rate by Objective IKDC score was 95\% (21 patients) who had an "A" score after 1 year from the operation. One patient didn't show any clinical improvement, however a repeating MRI revision arthroscopy showed good results of the repair. The Objective IKDC score in 3 years follow up was an "A" in all of the 13 patients who had completed the follow up.

The subjective IKDC and Lysholm scores were improved statistically after the operation. The subjective IKDC score was improved from a mean of 45 before the operation to a mean of 86 after 1 years from the operation ( $p$ value $<0.001$ ) to a 93 after 3 years from the operation (in the 13 patients that had completed the follow up). The Lysholm score was improved from a mean of 63 to 91 after 1 year from the operation ( $p$ value $<0.001)$ and to 93 in 3 years from operation (in 13 patients).

It is mention to say that 9 patients had a revision arthroscopy during the 3 years follow up. All of them underwent partial meniscectomy with removal of the suture. Three of these revision arthroscopies were performed during the first year (one after infection and two because of knee pain).

\section{Discussion}

The meniscus has a crucial role in shock absorption, load transmission, joint stability, and nutrition to the articular cartilage of the knee [12,13-18]. Preservation of meniscal tissue is ideal and the techniques for meniscal repair have been developing during the last decades. The overall clinical success rate with the Rapid Loc suture in our study was $95 \%$ for one-year follow-up, which is consistent with another report of the device [12]. By the 3 years follow up the clinical success was $59 \%$ of 13 patients that fulfill the follow up. Although the known criticism regarding the RapidLoc device including the slit formation through the cartilage and the inability to tension the knots after placement, all of them returned to their former activity level, and still doing so since the operation. The younger patients achieved better scores although not statistically significant.

Although in other studies, concurrent ACL reconstruction improved the outcome of meniscus repair; in our study no difference was seen. In conclusion, although this study was done a decade ago, before the presence of the third-generation devices, the Rapid Loc meniscal repair system offers many advantages as the "All-inside" rigid implants. The meniscus preservation with the continuation of the patients with their previous activity level is the prove of that. As mentioned above, 13 young patients (out of 22) live with their preserved meniscus for many years and enjoy its benefit.

During the years we also progress to use the fourth generation devices but this study showed that the device was safe and effective technique for meniscal repair. It has a high success rate and low complications. We recommend the preservation of the meniscus whenever possible using the existing devices system to repair peripheral tears.

\section{References}

1. Scott G, Jolly B, Helming C (1986) Combined posterior incision and arthroscopic intraarticular repair of the meniscus: An examination of factors affecting healing. J Bone Joint Surg Am 68: 847-861.

2. Andrew L Hass, Anthony A Schepsis, Joshua Hornstein, Cory M. Edgar (2005) Meniscal repair using the fast-fix all-inside meniscal repair device: Arthroscopy 21: 167-175.

3. DeHaven K, Amoczky SP, (1994) Meniscal repair Part I: Basic science, indications for repair, and open repair. J Bone Joint Surg Am 76: 140-152.

4. Warren RF (1990) Meniscectomy and repair in the anterior cruciate ligament-deficient patient. Clin Orthop 252: 55-63.

5. Tsai AM, McAllister DR, Chow S, Young CR, Hame SL (2004) Results of meniscal repair using a bioabsorbable screw. Arthroscopy 20: 586-590.

6. Morgan CD (1991) The "all inside" meniscus repair: Arthroscopy 7: 120-125.

7. Morgan CD (1993) The "all inside" arthroscopic meniscus repair: Techniques in orthopedics 8: 105-112.

8. Mulhollan JS (1993) Meniscus repair. In: Parisien JS, ed. Techniques in therapeutic arthroscopy. New York NY: Raven 21.

9. Reigel CA, Mulhollan JS, Morgan CD (1996) Arthroscopic all-inside meniscus repair: Clin sports med 15: 483-498.

10. David R Diduch, Kornelis A Poelstra (2003) The evolution of all-inside meniscal repair: Operative techniques in sports medicine 11: 83-90.

11. F Alan Barber, David A Coons, Michel Ruiz-Suarez (2006) meniscal repair with the Rapidloc meniscal repair device: Arthroscopy 22: 962-966.

12. Quinby JS, Golish SR, Hart JA, Diduch DR (2006) All-inside meniscal repair using a new flexible, tensionable device. Am J Sports Med 34 $1281-1286$ 
Citation: Ginesin E, Dreyfuss D, Stahl I, Peskin B (2017) All Inside Meniscal Repair: Three Years Follow up. Sports Injr Med: JSIMD-118. DOI: 10.29011/JSIMD$118 / 100018$

13. Fukubayashi T, Kurosawa H (1980) The contact area and pressure distribution pattern of the knee. A study of normal and osteoarthrotic knee joints. Acta Orthop Scand 51: 871-879.

14. Fairbank TJ (1948) Knee joint changes after menisectomy. J Bone Joint Surg Br 30: 664-670.

15. Henning CE, Lynch MA, Yearout KM, Vequist SW, Stallbaumer RJ, et al. (1990) Arthroscopic meniscal repair using an exogenous fibrin clot. Clin Ortop Relat Res 252: 64-72.
16. Johnson MJ, Lucas GL, Dusek JK, Henning CE (1999) Isolated arthroscopic meniscal repair: A long-term outcome study (more than 10 years). Am J Sports Med 27: 44-49.

17. Krause WR, Pope MH, Johnson RJ, Wilder DG (1976) Mechanical changes in the knee after menisectomy. J Bone Joint Surg Am 58: 599-604.

18. Levy IM, Trozilli PA, Gould JD, Warren RF (1989) The effect of lateral menisectomy on motion of the knee. J Bone Joint Surg Am 71: 401-406. 